\title{
Stiffness in total knee arthroplasty
}

\author{
Alfredo Schiavone Panni - Simone Cerciello • \\ Michele Vasso $\cdot$ Mario Tartarone
}

Received: 3 September 2008 / Accepted: 3 June 2009/Published online: 7 July 2009

(C) Springer-Verlag 2009

\begin{abstract}
Stiffness is a relatively uncommon complication after total knee arthroplasty. It has been defined as a painful limitation in the range of movement (ROM). Its pathogenesis is still unclear even if some risk factors have been identified. Patient-related conditions may be difficult to treat. Preoperative ROM is the most important risk factor, but an association with diabetes, reflex sympathetic dystrophy, and general pathologies such as juvenile rheumatoid arthritis and ankylosing spondylitis has been demonstrated. Moreover, previous surgery may be an additional cause of an ROM limitation. Postoperative factors include infections, arthrofibrosis, heterotrophic ossifications, and incorrect rehabilitation protocol. Infections represent a challenging problem for the orthopaedic surgeon, and treatment may require long periods of antibiotics administration. However, it is widely accepted that an aggressive rehabilitation protocol is mandatory for a proper ROM recovery and to avoid the onset of arthrofibrosis and heterotrophic ossifications. Finally, surgeryrelated factors represent the most common cause of stiffness; they include errors in soft-tissue balancing, component malpositioning, and incorrect component
\end{abstract}

A. Schiavone Panni - S. Cerciello - M. Vasso - M. Tartarone Science of Health Department, Molise University,

Campobasso, Italy

e-mail: a.schiavone@iol.it

M. Vasso

e-mail: michelevasso@alice.it

S. Cerciello $(\square)$

Viale Parioli 40, 00197 Rome, Italy

e-mail: simo.red@tiscali.it sizing. Although closed manipulation, arthroscopic and open arthrolysis have been proposed, they may lead to unpredictable results and incomplete ROM recovery. Revision surgery must be proposed in the case of welldocumented surgical errors. These operations are technically demanding and may be associated with high risk of complications; therefore they should be accurately planned and properly performed.

Keywords Stiffness - Arthrofibrosis .

Closed manipulation - Arthroscopic arthrolysis .

Revision surgery $\cdot$ Knee prosthesis

\section{Introduction}

Prosthetic surgery has evolved as a safe and satisfactory procedure in the treatment of degenerative pathologies involving the knee joint. More than 350,000 primary replacements and 29,000 revisions were performed in the United States in 2002 [1]. The increasing number of revision procedures has led to a better understanding of the different postoperative complications. Stiffness is one of the most complex, both in terms of pathogenesis and treatment as it represents a frustrating problem for surgeon and patient. Stiffness is defined by a range of movement (ROM) limitation often associated with persistent pain. Normal knee ROM ranges from 0 to $140^{\circ}$, while a ROM from 0 to $110^{\circ}$ after total knee arthroplasty (TKA) can be defined as a good result. No consensus is present in literature about the precise definition of stiffness. Stiffness has been defined by Kim et al. [2] as a flexion contracture $>15^{\circ}$ and a maximum flexion $<75^{\circ}$, by Yercan et al. [3] as postoperative ROM smaller than $10-90^{\circ}$, by Nicholls and Dorr [4] as a flexion contracture $\geq 20^{\circ}$ and a maximum 
flexion $\leq 45^{\circ}$, by Scranton [5] as a maximum flexion $\leq 85^{\circ}$, and by Christensen [6] as maximum ROM $\leq 70^{\circ}$.

The situation is extremely variable even in terms of epidemiology: Kim et al. [2] report an incidence of $1.3 \%$, Yercan et al. [3] of 5.3\%, and Gandhi et al. [7] of 3.7\%.

We believe a TKA should guarantee a postoperative ROM of $0-120^{\circ}$, even if a lower ROM $\left(5-95^{\circ}\right)$ is sufficient for most daily living activities. Scuderi [8] noted that a flexion of $65^{\circ}$ is required to walk on a flat ground, of $70^{\circ}$ to arise from a chair and of $90^{\circ}$ to descend stairs. Laskin and Beksac [9] stated that walking on a level surface requires $45-55^{\circ}$ of flexion, ascending or descending stairs about $85^{\circ}$, standing from a chair usually $95^{\circ}$, while kneeling requires $125^{\circ}$. These data are not always correlated with patient satisfaction: postoperative flexion of $80^{\circ}$ is unsatisfactory in the case of preoperative flexion of $120^{\circ}$, whereas it may be acceptable in the case of preoperative flexion of $60^{\circ}$.

\section{Materials and methods}

Due to the complexity of this issue, we performed a literature review to have a better comprehension of the problem. We searched the generally accepted PubMed index with the following keywords: stiffness, arthrofibrosis, total knee arthroplasty, and total knee replacement.

The combination of stiffness and total knee arthroplasty resulted in 139 articles, the one of arthrofibrosis and total knee arthroplasty resulted in 34 articles, while the combination of arthrofibrosis and total knee replacement resutled in 35 articles. Finally, the association between stiffness and total knee replacement resulted in 140 articles. The available abstracts were then evaluated, and the articles that correlated the best with the issue were examined.

From the analysis of these articles, some risk factors and treatment guidelines emerged. Although the pathogenesis was not completely clarified, we identified preoperative, intraoperative, and postoperative risk factors for stiffness. Moreover, according to the degree of ROM limitation, pathogenesis, and delay from the onset of the symptoms, some treatment options can be proposed: closed manipulation, arthroscopic or open debridement, and revision surgery.

\section{Pathogenesis}

General factors

In the pathogenesis of a stiff knee after TKA, several factors such as patient-related factors, surgical technique errors, and postoperative complications should be considered.

Patient factors include preoperative ROM, preoperative diagnosis, body habits, and patient personality [8]. Preoperative ROM reduction is the most important risk factor for postoperative stiffness [10]. This limitation may be the result of extensor mechanism and capsule contracture, posttraumatic arthrosis, and previous septic arthritis. Different pathologies such as juvenile rheumatoid arthritis and ankylosing spondylitis seem to play a role in the development of postoperative stiffness. Moreover, Jordan et al. [11] report the results of 17 total knee arthroplasties in patients affected by poliomyelitis, showing two cases of postoperative stiffness. Body mass index is not strictly related with postoperative ROM, especially in the case of trunk obesity and thin legs. On the other hand, flexion reduction may be caused by posterior impingement due to subcutaneous fat. Moreover, a correlation with general pathologies such as diabetes $[7,11,12]$ and lung disease [11] has been shown. Comorbidities such as heart disease and hypertension seem not to affect the final outcome after total knee arthroplasty, nor do alcohol, preoperative narcotic, and nonsteroidal anti-inflammatory drug use [11]. Smoking, however, has a negative influence on postoperative ROM [11]. Previous surgery of the involved knee is an important risk factor: Scranton [5] reports that $85 \%$ of the patients with a stiff knee after TKA have had previous surgery or diabetes mellitus (Fig. 1). Age seems to play a controversial role; some authors reported a higher incidence of postoperative stiffness in younger patients undergoing TKA $[11,13]$. Work status has an influence on postoperative results: retired patients were 1.5 times less likely to have stiff/poor result, whereas patients with disability or those not working due to knee pain were 5.8 times more likely to have stiff/poor result [11].

Reflex sympathetic dystrophy is a particular condition characterized by knee pain and stiffness. It is present in $0.8 \%$ of patient undergoing TKA [14]. Diagnosis is difficult as common exams are often normal. Patients describe delayed functional recovery, severe pain, vasomotor alterations, and trophic changes. Skin hypersensitivity, low local temperature, and hyperhydrosis may be present.

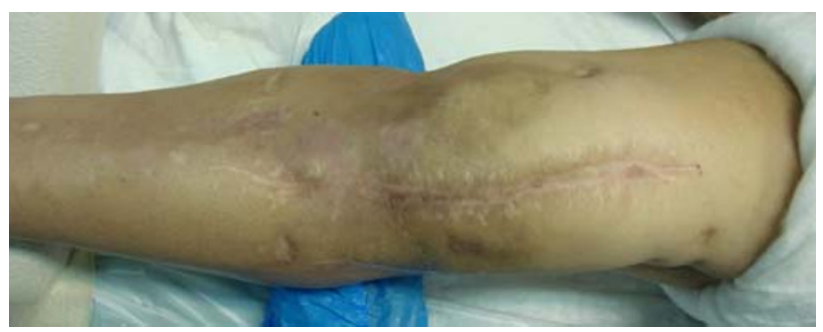

Fig. 1 Previous surgery is a risk factor in stiffness development 
Flexion is more often affected than extension. Patellar osteoporosis may be present in X-rays, and bone scan shows a diffuse hyperfixation. In the case of early diagnosis (less than 6 weeks), gentle mobilizations and anti-inflammatory drugs are sufficient; conversely, in the case of late diagnosis (more than 6 weeks), lumbar sympathetic block may be necessary. This procedure is both diagnostic and therapeutic, since in the case of nonresponse, the diagnosis should be reconsidered. Several blocks may be required for the resolution of complete symptoms.

Finally, a positive attitude to perform rehabilitation correctly plays an important role in functional recovery: depressed patients and patients with low threshold for pain are at risk of difficult rehabilitation [15].

\section{Surgical errors}

Most frequent causes involved in the development of postoperative stiffness are related to surgical errors. These include errors in soft-tissue balancing, component malpositioning, and incorrect component sizing [6,8].

In the case of cruciate-retaining prosthesis, a too tight posterior cruciate ligament limits the degree of maximum flexion, while a too loose posterior cruciate ligament causes an excessive femoral anterior translation with an impingement of the extensor mechanism. In the case of fixed varus deformity greater than $15-20^{\circ}$, the posterior cruciate ligament is involved in the deformity, and its resection or at least a wide release is necessary [16, 17].

Excessive femoral component hyperflexion may cause potential block to full extension and excessive tension on the medial and lateral retinacula; excessive hyperextension may lead to anterior notching and limited flexion [9]. Traditional intramedullary guides align the femoral component to the anatomic axis which is slightly hyperflexed if compared to the lower limb axis. Excessive tibial slope increases the posterior space leading to a loose PCL in cruciate-retaining implants. Insufficient tibial slope creates a smaller posterior space with flexion limitation. Most authors suggest the amount of posterior slope resulting from bony resections and insert slope should match the preoperative articular slope [9].

Excessive "patellofemoral" thickness is the consequence of an excessively anterior implant (in the case of posterior reference), an insufficient patellar resection, or an oversized femoral component (Fig. 2). An increase of more than $4 \mathrm{~mm}$ is considered pathologic [18].

Incorrect joint-line height is a potential cause of ROM limitation. Lowering the joint line leads to a patella alta and to tight retinacula causing pain and limited flexion. Raising the joint line leads to a patella baja and to an impingement with the polyethylene insert causing anterior knee pain and limited knee flexion [19] (Fig. 3).

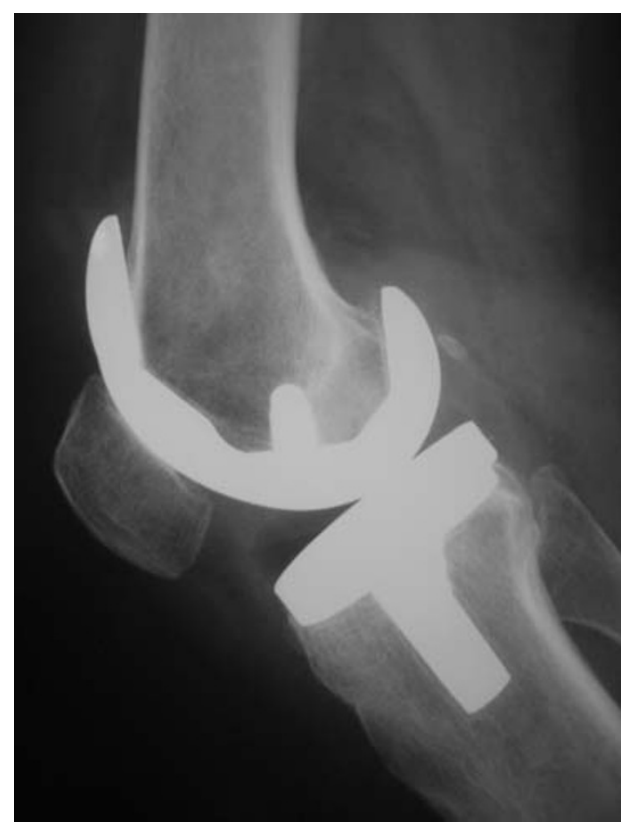

Fig. 2 Oversizing in the femoral component causes increase in patella contact stresses with persistent anterior knee pain and ROM reduction

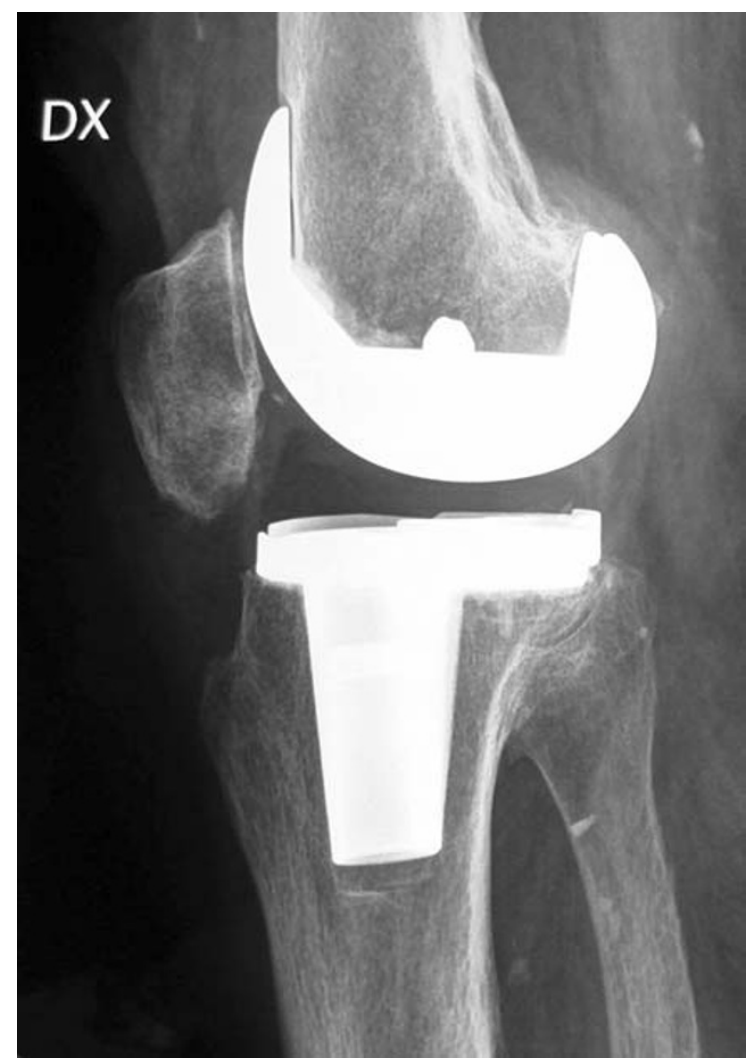

Fig. 3 A patella baja may lead to an anterior impingement with the polyethylene insert and flexion limitation 
Errors in bony resection may cause flexion-extension mismatch with ROM reduction.

A proper distal femoral cut allows a good soft-tissue balance in extension. An insufficient posterior condyles resection may cause excessive tension on the collateral ligaments and PCL (if preserved) with consequent limited flexion. The possible solutions are a smaller femoral component (small anteroposterior diameter) or lowering the distal femoral cut using a thinner polyethylene. On the other hand, an excessive posterior condyle resection leads to a loose knee in flexion; a distal femoral recut using a thicker polyethylene would balance the knee both in flexion and extension.

Concerning the distal femoral resection, an insufficient resection leads to a stiff knee in extension. A distal femoral recut and posterior capsule release would be necessary to solve this problem. An excessive distal femoral resection leads to a loose knee in extension; a smaller femoral component (smaller antero-posterior diameter) with a thicker polyethylene allows a balance in flexion and extension. Posterior osteophytes often cause an extension contracture, and accurate removal is always necessary. The previous factors limit intraoperative range of motion, and this situation becomes even worse in the postoperative period. Other errors are difficult to detect intraoperatively, but usually become evident as the rehabilitation goes on. For example, the malrotation of the femoral component is difficult to detect during surgery but may cause patellofemoral kinematics alterations and microtraumatisms that lead to an inflammatory reaction and to an arthrofibrosis.

Finally, prosthesis fixation seems to play a less important role in the development of postoperative stiffness: Harvey et al. [20] do not report any difference between cemented and uncemented implants. Duffy et al. [21] report a slightly better postoperative flexion in the case of cementless implants $\left(102 \pm 18^{\circ}\right.$ vs. $\left.100 \pm 19^{\circ}\right)$, although the only case of postoperative stiff knee was found in this group. Dodd et al. [22] report a postoperative ROM of 108 and $102^{\circ}$ in the cemented and uncemented groups, respectively, at 6 months. By the 2 -year FU period, the ROMs were equal. Three knees required manipulation under anesthesia (both knees of a rheumatoid patient and one knee in the cemented group) [22].

\section{Postoperative factors}

Infections may be a cause of ROM limitation. Usually they appear with few symptoms such as a difficult and slow rehabilitation. Systemic involvement with high temperature is uncommon. Pain is not always present. For these reasons, a postoperative stiff knee should always be considered as infected. Staphylococcus epidermidis is usually responsible for intense joint effusion and scar-tissue production. Arthrofibrosis is the most common cause of stiffness with an incidence ranging from 1.2 to $17 \%$ according to literature. It is caused by progressive production of abundant scar tissue between extensor mechanism and anterior femoral cortex with suprapatellar pouch obliteration, and medial and lateral gutters scarring down. No predisposing factors have been identified even if prolonged postoperative immobilization seems to be involved.

Aggressive rehabilitation is thus recommended and should start as soon as possible; the CPM (continuous passive motion) device allows a faster flexion recovery. Its role is essential in the first 3 months after surgery; later, it becomes almost useless. Joint haematomas may slow down rehabilitation and may develop an abundant fibrous tissue. Heterotrophic ossifications may cause postoperative stiffness; their incidence ranges from 3.8 [23] to $26 \%$ [24], and no correlation exists between their localization and the degree of stiffness. They are more frequent in the suprapatellar pouch and supracondylar region (up to $26 \%$ of the patients) [23, 24]. They are more frequent after wide surgical exposition, closed manipulation, and in patients with increased vertebral bony density. Some authors have noted an influence of Coumadin dosage after surgery [25]. Treatment consists of excision and physical therapy.

Sometimes stiffness is not caused by the knee itself but rather by problems affecting other joints. Clinical outcome can be influenced by hip or spine disorders. Hip flexion contracture or dorsal-lumbar kiphosis can lead to knee flexum. Their evaluation and treatment is important and should be performed before knee surgery. Moreover, in the evaluation of a stiff knee, several neurological or muscular pathologies should be considered [26]. Finally, proper rehabilitation is mandatory to obtain satisfactory articular motion: intensive passive motion and pain control must be performed immediately after surgery. Conversely, a too aggressive rehabilitation may lead to an inflammatory reaction with persistent pain and joint contracture.

\section{Treatment}

Prevention is the best way to treat a stiff knee. However, correct diagnosis is the first step to start a correct treatment. Beyond the special cases such as arthrofibrosis, heterothopic ossifications, and reflex sympathetic dystrophy whose treatment has been previously discussed, general treatment consists of the following options: manipulation, artrolysis (open or arthroscopic), and revision surgery.

\section{Closed manipulation}

This is a debated option, and its timing is critical. This procedure seems to accelerate ROM recovery in patients 
with slow and difficult rehabilitation rather than allowing for a greater final ROM. Some authors suggest a closed manipulation in all patients not reaching $90^{\circ}$ of flexion on the 10th day after surgery [27], others wait for at least 2 weeks [28]. The incidence of closed manipulations is therefore 54 and 23\%, respectively. Esler et al. [29] suggest a closed manipulation in all patients not reaching $80^{\circ}$ of flexion regardless of the delay from the surgical procedure. In general, the commonly accepted indication is a postoperative flexion inferior to $90^{\circ}$ at 6 weeks, and the earlier this manipulation is performed, the more satisfactory is the result as showed by Daluga [30]. In the case of a too early manipulation, a risk of skin and soft-tissue damage is present; thus, a delay of at least 3 weeks from the surgical procedure is indicated. Its aim is to strip the fibrous bands that originate in the suprapatellar pouch in the case of incorrect rehabilitation. These bands become stronger and stiffer beyond 6 weeks with increased risk of complications such as patellar or femoral fracture or extensor mechanism rupture in the case of closed manipulation.

Manipulation should be performed under general or regional anaesthesia to provide adequate muscle relaxation, reducing the risk of complications. Once the effective ROM is recorded, a gentle force is applied to force the maximum flexion; mobilization of the patella is indicated, as it destroys the adherences in the suprapatellar pouch. This maneuver should be repeated several times for a complete lysis of fibrous bands. Postoperative management consists of continuous passive motion set to the maximum flexion and extension obtained. Pain control is essential: an epidural catheter is set in place for $24-48 \mathrm{~h}$. Some authors stress the importance of pain management during rehabilitation and report that proper pain control reduces the necessity of closed manipulation from 9 to $1 \%$ [31].

Results seem satisfactory in the case of correct indications and precise technique: Yercan reports a ROM improvement from 67 to $117^{\circ}$ [32], Pariente et al. [33] from 71 to $102^{\circ}$ with his modified technique, while Scranton [5] reports an average gain of 36 and $35^{\circ}$ if the manipulation is performed within 12 weeks and beyond this limit, respectively.

\section{Surgical treatment}

When the correct rehabilitation protocol is performed with no results for at least 6-12 weeks, no more improvements should be expected and surgical treatment should be considered.

\section{Arthroscopic arthrolysis}

Arthroscopic arthrolysis consists of lysis of direct adherences and is indicated in the case of arthrofibrosis in patients with a difficult rehabilitation. The ideal indication is a painless, stiff knee that has not improved after 3-6 months of conservative treatment, as reported by Court [34]. On the other hand, poor results are reported in painful stiff knee; this seems to be a consequence of wrong diagnosis [35].

This procedure guarantees a wide access to the suprapatellar pouch thus allowing good flexion recovery, while the access to the posterior part of the joint is extremely difficult or impossible. Thus, it is less effective in the case of extension lag. A PCL release or sacrifice is possible and is indicated in cruciate-retaining implants when a tight PCL is found. Its section leads to an immediate flexion gap increase and confirms that the diagnosis was correct. Foreign body removal (cement) is also possible under arthroscopy. Classic portals are sufficient in the majority of cases; if patellofemoral fibrosis is expected, then additional superior portals (medial and lateral) may be indicated. Synovial and scar samples are taken for a microbiological and histological analysis.

Jerosch describes a standard technique that consists of release of all fibrous bands in the suprapatellar pouch, re-establishing of the medial and lateral gutters, patellar release, and removal of remaining meniscal tissue or anterior cyclops. He reports a mean postoperative flexion of $119^{\circ}$ and a decrease in extension lag from 27 to $4^{\circ}$ [36].

Results after arthroscopic arthrolysis are rather controversial in the literature. Bocell et al. [35] report an increase in postoperative ROM in $43 \%$ of the patients, Williams et al. [37] an average increase in ROM of $30.6^{\circ}$, while Mont et al. [38] report an average improvement of $31^{\circ}$ in $94 \%$ of the patients. Best results are obtained in the case of isolated patellofemoral fibrosis. As for the closed manipulation, best results are obtained in the case of early procedure. Most authors recommend not delaying beyond 6 months to avoid the scar tissue becoming strong and resistant. The importance of an aggressive postoperative protocol consisting of continuous passive motion and proper pain control after arthroscopic arthrolysis is now widely accepted [39]. In the case of severe ROM limitation, arthroscopic treatment alone is less effective, as demonstrated by Scranton [5], who noted worse results in the case of $\mathrm{ROM} \leq 60^{\circ}$.

Open arthrolysis

Keeney et al. [40] recommend open arthrolysis in the case of a severe ROM limitation after TKA with no component malposition and after a proper conservative treatment performed for 6 months after surgery. It may be associated with polyethylene insert or patellar component exchange. Complete exposition may be difficult due to adherences, 
and posteromedial corner release facilitates this maneuver. If patellar eversion is impossible, then tibial tubercle osteotomy or quadriceps tendon snip or VY plasty may be indicated. Suprapatellar pouch and medial and lateral gutters should be explored to look for fibrous bands. Posterior release is indicated to improve extension, while a 1- to $2-\mathrm{cm}$ proximal transfer of the tibial tuberosity increases knee flexion and raises the patella with a minimal loss of quadriceps strength. Scuderi [8] proposes the quadriceps snip and tendon lengthening to improve maximum flexion with no extension or strength loss. Patellar resection is indicated in the case of overstuffing. In the case of flexion contracture, polyethylene downsizing is indicated.

Unfortunately, only a few reports are available in the literature concerning the results of open arthrolysis in the treatment of a stiff knee. In a Sofcot series, flexion and extension gains were 20 and $18^{\circ}$, respectively [41]; Hutchinson reported an increase from 55 to $91^{\circ} 6$ months after open arthrolysis [42]; and Pretzsch showed an increase in knee flexion from 46 to $90^{\circ}$ and a decrease in flexion contracture from 11 to $7^{\circ}$ [43]. In contrast, Babis reports poor results in the case of open arthrolysis and polyethylene exchange for stiff knees with fixed and wellaligned prosthetic component [44].

\section{Revision surgery}

In the case of documented surgical errors, any attempt to improve ROM must consider the resolution of these errors, making revision surgery necessary. Accurate analysis of these errors is essential to plan the revision correctly and to evaluate the expected benefits. In fact, functional results are usually unpredictable and ROM recovery may be poor. The incision should consider the skin contracture and fibrosis; scar excision should be avoided; and in the case of multiple incisions, the most lateral should be preferred. As previously mentioned, patellar tendon avulsion may occur due to its shortening, and fibrosis, tibial tuberosity osteotomy, quadriceps snip, or VY plasty are often required. Scar-tissue removal is performed in the same manner as in the case of open arthrolysis. Component stability, evidence of polyethylene wear, and patello-femoral track are carefully examined. Component removal may lead to bone stock loss especially in the case of uncemented implants, requiring wedge and augmentations to address this loss.

Great care should be taken with the patella, which is a cause of stiffness requiring revision surgery in $55 \%$ of the cases as shown by Bonnin et al. [45]. Patellar thickness is measured, since it is a common cause of stiffness when it is excessive. In the case of thickness greater than $26 \mathrm{~mm}$ in males and $24 \mathrm{~mm}$ in females, a resection is indicated [46]. Soft-tissue balance is performed both in flexion and extension, and minimal symmetrical laxity in extension should be preferred to stiff knee. Considering the frequent recurrence of stiffness, some authors prefer to obtain a hyperextension of $5^{\circ}$ at the end of revision surgery [47]. It has been proved that a tibial overresection of $1 \mathrm{~mm}$ increases the extension space by $4^{\circ}[48]$.

The problem of joint-line level is still debated. Epicondiles are a good anatomical reference in joint-line restoration as described by Griffin. In unaffected knees, the medial epicondile is $27.4 \pm 2.9$ and $29.7 \pm 2.7 \mathrm{~mm}$ from the joint line in coronal and axial planes, respectively. Lateral epicondile is $24.3 \pm 2.6$ and $25.0 \pm 2.6 \mathrm{~mm}$ from the joint line in coronal and axial planes, respectively [49]. Excessive joint-line elevation leads to a patella baja and to loose collateral ligaments in flexion. Figgie et al. [19] state that an elevation up to $8 \mathrm{~mm}$ has no major consequences for the postoperative result. Unfortunately, in revision surgery, the epicondiles are not always clearly detectable; thus, the evaluation of the joint-line height may be difficult. Therefore, the distance from the inferior pole of the patella to the joint line has been proposed as a measure, and values greater than $1 \mathrm{~cm}$ are necessary to restore a proper height [19]. Component malpositioning in the frontal, sagittal, and coronal plane should be corrected to avoid stiffness recurrence. Frontal malalignments and rotational tibial and femoral errors should be corrected, as they affect both patellofemoral kinematics and soft-tissue balance. Postoperative results are unpredictable both in terms of articular recovery and clinical improvement.

Haidukewych et al. [50] in a series of revisions for stiffness report a ROM improvement from 40 to $73^{\circ}, 66 \%$ of satisfactory results, and a further revision rate for stiffness of $25 \%$. Dorr and Nicholls report fair and unpredictable results in the case of revision. Positive prognostic factors were malpositions and previous osteoarthrosis, and negative factors were patella baja and previous rheumatoid arthritis [4]. Nelson et al. [10] report an improvement of flexion contracture from 11.3 to $3.2^{\circ}$ and flexion improvement from 65.8 to $85.4^{\circ}$. A postoperative ROM improvement was present in $93 \%$ of the patients.

Keeney compared the results of a limited approach (softtissue release and tibial insert downsizing) with those of a complete revision surgery. In the first group, there was an improvement in postoperative ROM $\left(25.7^{\circ}\right)$, clinical score (37.8 points), and functional score (20.8 points). In the second group, the improvement in postoperative ROM was $17.9^{\circ}$, in functional score was 3.6 points, while there was a decrease of 1.0 point in clinical score [40].

Bonnin [45] reported a ROM increase of $41^{\circ}$ with associated symptoms improvement. In a SOFCOT series, there was a flexion improvement of $35^{\circ}$ with an average postoperative flexion of $83^{\circ}$; moreover, an improvement in flexion contracture and pain score was noted [41]. 


\section{Discussion}

Stiffness is a frustrating complication after total knee arthoplasty. Its precise definition is still debated [2, 6]. Several factors contribute to its development and may be divided into preoperative, intraoperative, and postoperative.

Preoperative factors include preoperative ROM, and previous knee surgery and general factors such as diabetes, lung diseases, and smoking [11]. Reflex sympathetic dystrophy is a particular situation characterized by pain and stiffness [14].

Depression may compromise postoperative rehabilitation protocol and may therefore lead to a poor result after total knee arthroplasty.

Intraoperative factors include soft-tissue balancing mismatch, component malpositioning, and incorrect implant sizing $[6,8]$. PCL management is crucial; its excessive tension is a source of flexion reduction. Moreover, joint-line variations may cause extensor mechanism unbalance and medial and lateral retinacula over-tension thus leading to ROM reduction and pain [19]. Tibial and femoral internal rotation affect both tibiofemoral and patellofemoral kinematics with inflammatory reaction and further arthrofibrosis.

Postoperative factors include arthrofibrosis and heterotopic ossifications and postoperative rehabilitation protocol. Thus, correct pain management in the first days after surgery seems mandatory to avoid these problems. Once the stiffness has been diagnosed, several options are available depending on its severity and cause and on the delay between the surgical procedure and the diagnosis. Closed manipulation is indicated in the first 2-3 weeks after surgery [27, 28]. It allows the fibrous bands that develop in the joint and suprapatellar pouch to be broken. In the case of correct indications, it guarantees satisfactory results [31-33]. Surgical options may be proposed after 6-12 weeks of a correct rehabilitation program. Arthroscopic arthrolysis is indicated in the case of stiff and painfree knees. It is a good option, since it is a minimally invasive procedure and permits lysis of fibrous bands, PCL release, and removal of loose bodies. Unfortunately, clinical results are controversial and its indication is limited in severe cases [35-38]. Open arthrolysis is a more invasive option, and it allows a wide access to the anterior and posterior aspects of the joint. Moreover, polyethylene insert exchange is possible. Clinical results are generally satisfactory, although few articles are available in literature [41-43].

Revision surgery is the most aggressive and technically demanding option. It is indicated in the case of well-documented surgical errors. Patellar thickness, height, and tracking are a common source of problems leading to stiff knee; therefore, they should be carefully evaluated [45]. Moreover, component malpositioning or oversizing should be corrected as should joint-line variations. Even in the case of a proper indication and precise surgical technique, results are sometimes unsatisfactory. Therefore, preoperatively patients should be carefully briefed on the unpredictable results following a revision procedure for stiffness.

Conflict of interest statement We certify that there is no actual or potential conflict of interest in relation to this article.

\section{References}

1. Mahomed NN, Barret J, Katz JN, Baron JA, Wright J, Losina E (2005) Epidemiology of total knee replacement in the United States medicare population. J Bone Joint Surg Am 87A(6):12221228

2. Kim J, Nelson CH, Lotke PA (2004) Stiffness after total knee arthroplasty: prevalence of complication and outcome of revision. J Bone Joint Surg 86-A:1479-1484

3. Yercan HS, Sugun TS, Bussiere C, Ait Si Selmi T, Neyret P (2004) Stiffness after total knee arthoroplasty. J Lyon Chir Genou. 11:327-336

4. Nicholls DW, Dorr LD (1990) Revision surgery for stiff total knee arthroplasty. J Arthroplasty 5:269-281

5. Scranton PE Jr (2001) Management of knee pain and stiffness after total knee arthroplasty. J Arthroplasty 16:428-435

6. Christensen CP, Crawford JJ, Olin MD, Vail TP (2002) Revision of stiff total knee arthroplasty. J Arthroplasty 17:409-415

7. Gandhi R, de Beer J, Leone J, Petruccelli D, Winemaker M, Adili A (2006) Predictive risk factors for stiff knees in total knee arthroplasty. J Arthroplasty 21(1):46-52

8. Scuderi GR (2005) The stiff total knee arthroplasty: casuality and solution. J Arthroplasty 4(Suppl 2):23-26

9. Laskin RS, Beksac B (2004) Stiffness after total knee arthroplasty. J Arthroplasty 4(suppl 1):41-46

10. Nelson CH, Kim J, Lotke PA (2005) Stiffness after total knee arthroplasty. J Bone Joint Surg 87-A(Supp1 Pt2):264-270

11. Jordan L, Klingman M, Sculco TP (2007) Total knee arthroplasty in patients with poliomyelitis. J Arthroplasty 22(4):543-548

12. Meding JB, Reddleman K, Keatng ME, Klay A, Ritter MA, Faris PM, Berend ME (2003) Total knee replacement in patients with diabetes. Clin Orthop 416:208-216

13. Parvizi J, Tarity TD, Steinbeck MJ, Politi RG, Joshi A, Purtill JJ, Sharkey PF (2006) Management of stiffness after total knee arthroplasty. J Bone Joint Surg 88-A(supp 4):175-181

14. Katz MM, Hungerford DS (1987) Reflex sympathetic dystrophy affecting the knee. J Bone Joint Surg 69-B:797-801

15. Fisher DA, Dickerman B, Watts MR, Davis K (2007) Looks good but feels bad: factors that contribute to poor results after total knee arthroplasty. J Arthroplasty 22(6 suppl 2):39-42

16. Laskin R (1996) Total knee replacement with posterior cruciate ligament retention in patients with a fixed varus deformity. Clin Orthop 331:29-34

17. Lombardi AV, Mallory TH, Fada RA, Hartman JF, Capps SG, Kefauver CA, Adams JB (2001) An algorithm for posterior cruciate ligament in total knee arthroplasty. Clin Orthop 392:75-87

18. Star MJ, Haufman KR, Irby SE, Colwell CW (1996) The effects of patellar thickness on patellofemoral forces after resurfacing. Clin Orthop 322:279-284

19. Figgie HE, Goldberg VM, Heiple KG, Müller HS, Gordon NH (1986) The influence of tibial-patellar location on function of the 
knee in patients with posterior stabilized condylar knee prosthesis. J Bone Joint Surg 68A:1035-1040

20. Harvey IA, Barry K, Kirby SPJ, Johnson R, Elloy MA (1993) Factors affecting the range of movement of total knee arthroplasty. J Bone Joint Surg 75B:950-955

21. Duffy GP, Berry DJ, Rand JA (1998) Cement versus cementless fixation in total knee arthroplasty. Clin Orthop 356:66-72

22. Dodd CAF, Hungerford DS, Krackow KA (1908) Total knee arthroplasty fixation. Comparison of the early results of paired cemented versus uncemented porous coated anatomic knee prosthesis. Clin Orthop 260:66-70

23. Harwin SF, Stein AJ, Stern RE (1993) Heterotopic ossifications following primary total knee arthroplasty. J Arthroplasty 8:113116

24. Furia JP, Pellegrini VD (1995) Heterotopic ossifications following total knee arthoroplasty. J Arthroplasty 10:413-419

25. McClelland SJ, Rudolf LM (1986) Myositis ossification following porous-ingrowth TK replacement. Orthop Rev 15:223-227

26. Dellon AL, Mont MA, Krackow KA (1996) Partial denervation for persistent neuroma pain after total knee arthroplasty. Clin Orthop 316:145-150

27. Shoji H, Yoshino S, Komagamine M (1987) Improved range of motion with the Y/S total knee arthroplasty system. Clin Orthop Relat Res 218:150-163

28. Fox JL, Poss R (1981) The role of manipulation following total knee replacement. J Bone Joint Surg Am 63(3):357-362

29. Esler CN, Lock K, Harper WM, Gregg PJ (1999) Manipulation of total knee replacements. Is the flexion gained retained? J Bone Joint Surg Br 81(1):27-29

30. Daluga D, Lombardi AV, Mallory TH, Vaughn BK (1991) Knee manipulation following total knee arthroplasty: analysis of prognostic variables. J Arthroplasty 6:119-128

31. Heuleu JN, Neyret $P$ (2002) Conduite à tenir devant les raideurs postopératoires du genou. Rev Chir Orthop Reparatrice Appar Mot 88(Suppl 5):1S27-1S51

32. Yercan HS, Sugun TS, Bussiere C, Ait Si Selmi T, Davies A, Neyret P (2006) Stiffness after total knee arthroplasty: prevalence, management and outcomes. Knee 13(2):111-117

33. Pariente GM, Lombardi AV, Berend KR, Mallory TH, Adams JB (2006) Manipulation with prolonged epidural analgesia for treatment of TKA complicated by arthrofibrosis. Surg Technol Int $15: 221-224$

34. Court C, Gauliard C, Nordin JY (1999) Technical aspects of arthroscopic arthrolysis after total knee replacement. Rev Chir Orthop Reparatrice Appar Mot 85(4):404-410

35. Bocell JR, Thorpe CD, Tullos HS (1991) Arthroscopic treatment of symptomatic total knee arthroplasty. Clin Orthop 271:125-134
36. Jerosch J, Aldawoudy AM (2007) Arthroscopic treatment of patients with moderate arthrofibrosis after total knee replacement. Knee Surg Sports Traumatol Arthrosc 15(1):71-77

37. Williams RJ, Westreich GH, Siegl J, Winsord RE (1996) Arthroscopic release of posterior cruciate ligament for stiff total knee arthroplasty. Clin Orthop 321:185-191

38. Mont MA, Seyler TM, Marulanda GA, Delanois RE, Bhave A (2006) Surgical treatment and customized rehabilitation for stiff knee arthroplasties. Clin Orthop Relat Res 446:193-200

39. Diduch DR, Scuderi GR, Scott WN, Insall JN, Kelly MA (1997) The efficacy of arthroscopy following total knee replacement. Arthroscopy 13(2):166-171

40. Keeney JA, Clohisy JC, Curry M, Maloney WJ (2005) Revision total knee arthroplasty for restricted motion. Clin Orthop 440:135-140

41. Letenneur J, Guilleux C, Gruber P, Dauty M (2001) Les reprises de prothèse totale du genou pour raideur. Rev Chir Orthop 87:S149-S151

42. Hutchinson JR, Parish EN, Cross MJ (2005) Results of open arthrolysis for the treatment of stiffness after total knee replacement. J Bone Joint Surg Br 87(10):1357-1360

43. Pretzsch M, Dippold A (1999) Results of surgical arthrolysis in treatment of restricted knee joint movement. Z Orthop Ihre Grenzgeb 137(4):334-339

44. Babis GC, Trousdale RT, Pagnano MW, Morrey BF (2001) Poor outcomes of isolated tibial insert exchange and arthrolysis for the management of stiffness following total knee arthroplasty. J Bone Joint Surg 83-A:1534-1536

45. Bonnin M, Deschamps G, Neyret P, Chambat P (2000) Les changements des prothèses totales du genou non infectées. Analyse des résultats a propos d'une série continue de 69 cas. Rev Chir Orthop Reparatrice Appar Mot 86:694-706

46. Barnes CL, Scott RD (1993) Patellofemoral complications of total knee replacement. Instr Course Lect 42:303-307

47. Ries MD, Badalamente M (2000) Arthrofibrosis after total knee arthroplasty. Clin Orthop 380:177-183

48. Shoemaker SC, Markolf KL, Finerman GAM (1982) In vitro stability of the implanted total condylar prosthesis. J Bone Joint Surg 64A:1201-1213

49. Griffin FM, Math K, Scuderi GR, Insall JN, Poilvache PL (2000) Anatomy of the epicondyles of the distal femur: MRI analysis of normal knees. J Arthroplasty 15(3):354-359

50. Haidukewych GJ, Jacofsky DJ, Pagnano MW, Trousdale RT (2005) Functional results after revision of well-fixed components for stiffness after primary total knee arthroplasty. J Arthroplasty 2:133-138 